Friedrich et al.: Compound eye evolution

\title{
Ancient default activators of terminal photoreceptor differentiation in the pancrustacean compound eye: The homeodomain transcription factors Otd and Pph13
}

\author{
Markus Friedrich ${ }^{1,2}$, Tiffany Cook $^{3,4}$, Andrew C. Zelhof ${ }^{5}$
}

1. Department of Biological Sciences

Wayne State University

Detroit, MI 48202, USA

2. Department of Anatomy and Cell Biology

Wayne State University School of Medicine

Detroit, MI 48201, USA

3. Center of Molecular Medicine and Genomics

Wayne State University School of Medicine

Detroit, MI 48201, USA

4. Department of Ophthalmology

Wayne State University School of Medicine

Detroit, MI 48201, USA

5. Department of Biology

Indiana University

Bloomington, IN 47405, USA

Corresponding Author:

Dr. Markus Friedrich

Department of Biological Sciences

Wayne State University

5047 Gullen Mall

Detroit, MI 48202

Email: friedrichwsu@gmail.com

Phone: 3135779612

Keywords: Photoreceptor, Rhabdomere, Orthodenticle, Pph13, Drosophila, Tribolium, Daphnia, Evolution, Development, cis-regulatory DNA, enhancer, transcription factor, eye, retina, Hazy, Munster, Rhodopsin Conserved Sequence I (RCSI) 


\section{ABSTRACT}

The origin of the Drosophila compound eye predates the ancestor of Pancrustacea, the arthropod clade that includes insects and Crustaceans. Recent studies in emerging model systems for pancrustacean development - the red flour beetle Tribolium castaneum and water flea Daphnia

pulex - have begun to shed light on the evolutionary conservation of transcriptional mechanisms found for the Drosophila compound eye. Here, we discuss the conserved roles of the transcription factors Otd and Pph13, which complement each other in two terminal events of photoreceptor differentiation: rhabdomere morphogenesis and transcriptional default activation of opsin gene expression. The synthesis of these data allows us to frame an evolutionary developmental model of the earliest events that generated the wavelength-specific photoreceptor subtypes of pancrustacean compound eyes. 


\section{The pancrustancean compound eye: evolutionary conservation and cellular organization}

Compound eyes represent one of the most frequently evolved eye types in animals [1]. Their particularly elaborate version in insects and crustaceans (Pancrustacea) ranks among the most capable and complex known visual organs [2]. Remarkably, the compound eyes of some arthropod fossils from the Cambrium parallel extant species in structural detail $[3,4]$. Thus, the sophistication of the compound eye in present day pancrustacean species dates back hundreds of millions of years. Comparative developmental genetic studies have now elucidated the first conserved gene regulatory mechanisms that characterize this ancient but highly advanced ground state.

The key characteristic of compound eyes is their assembly from serially homologous visual subunits, called ommatidia. In most pancrustacean species, the core of a single ommatidium consists of 8 photoreceptor cells, 4 cone cells, and 2 primary pigment cells (Fig. 1) [5]. Surrounding this core, a more variable set of interommatidial pigment cells provides the optical insulation of individual ommatidia, while the cone cells and primary pigment cells form the individual lenses of each ommatidium. This system focuses light from a specific point of the environment on the underlying photoreceptors, which translate the photic information on the environment into neural signals to the brain.

The light capture takes place in a specialized apical membrane compartment of the photoreceptors, the rhabdomere, which houses the light-sensitive, seven-transmembrane Gprotein coupled opsin receptor proteins and the downstream phospholipase C-based phototransduction machinery. In most cases, a single photoreceptor specifically expresses one of several opsin genes, which differ in wavelength-sensitivity (WS), falling into three subfamilies: green or long wavelength-sensitive (LWS), blue or short wavelength-sensitive (SWS-B), and ultraviolet short wavelength-sensitive (SWS-UV) [6].

Together with structural features, the expression of specific opsin paralogs defines distinct subtypes among the eight photoreceptors of a single ommatidium. Some of these subtypes are deeply conserved. This includes the dichotomy between outer vs inner photoreceptors. The six outer photoreceptors R1-R6 are often characterized by rhabdomeres that span the entire depth of the retina and are arranged around the two inner photoreceptors R7 and 
Friedrich et al.: Compound eye evolution

R8. The rhabdomeres of the latter are centrally-positioned, with that of the R7 photoreceptor usually located distally to that of the R8 photoreceptor. As recently reviewed in new molecular detail [7], these basic photoreceptor subtypes have further diversified in lineage- or speciesspecific ways. Thus, while uniform from the outside due to conserved ancient traits in cellular

organization [8,9], pancrustacean compound eyes are also highly diverse in their complex neural organization, providing excellent opportunities for studying the developmental basis of the adaptive evolution of receptor diversity. Fundamental insights into this issue have been gained through the progress in understanding the molecular regulation of rhabdomere formation and differential opsin expression in Drosophila [10,11] and functional comparative studies in a broader range of pancrustacean model systems.

\section{Opsin expression, photoreceptor subtypes, and ommatidial subtypes in fruit flies, flour}

\section{beetles, and water fleas}

In addition to Drosophila, studies of compound eye development have been initiated in emerging pancrustacean model species that include the red flour beetle Tribolium castaneum [12], the grasshopper Schistocerca americana [13], the two-spotted cricket Gryllus bimaculatus [14,15], and, most recently, the water fleas Daphnia pulex and Daphnia magna [16]. Not surprisingly, these models differ in many visually-relevant features, given their diversified visual ecologies. Water fleas, for instance, are cyclopic, possessing only a single median compound eye in contrast to the typical pair of lateral compound eyes in most pancrustaceans (Fig, 2). Moreover, the cyclopic water flea compound eye consists of less than 20 ommatidia. This contrasts with close to 100 ommatidia in each of the two lateral compound eyes of Tribolium and about 750 ommatidia per compound eye in Drosophila (Fig. 2) [17-19]. Further remarkable is the that this variation in eye size correlates very little with the diversity of opsin genes expressed in the eyes of these species.

Only two opsin genes, for instance, are expressed in the Tribolium compound eye, with a singleton LWS opsin present in all photoreceptors, and a singleton SWS-UV opsin selectively expressed in the inner R7 photoreceptors (Fig. 2). The resulting co-expression of two opsins in the R7 photoreceptors is a derived exception to the 'one-receptor' rule typically required for 
Friedrich et al.: Compound eye evolution

wavelength discrimination in visual systems [20] and likely reflects the crepuscular ecology of Tribolium [21].

Astoundingly, the small-eyed Daphnia represents a much more complex model for differential opsin expression. One SWS-UV, three SWS-B, and 25 LWS opsins have identified from its genome [16,22]. While our understanding of the visual organization of Daphnia is still very limited, it is reasonable to speculate that this diversity of Daphnia opsins reflects the dynamic spectral characteristics of the freshwater environments to which this species adapted [23]. Physiological evidence suggests that, like in Drosophila, the Daphnia opsins are differentially expressed among photoreceptors within individual ommatidia and possibly in different combinations across the eye $[16,19,24]$. Consistent with this, recent molecular studies revealed that specific LWS opsins and the singleton SWS-UV opsin are indeed differentially expressed [22]. Moreover, there is molecular evidence that temporal and environmental variables impact the differential expression of Daphnia opsin genes [16]. Notwithstanding these first insights, presence and organization of photoreceptor subtypes and ommatidial subtypes in the Daphnia compound eye await to be elucidated.

In comparison to Daphnia, Drosophila expresses a moderate number of five opsin paralogs in the compound eye: two LWS (Rh1/ninaE and Rh6), one SWS-B (Rh5), and two SWS-UV opsins (Rh3 and Rh4) (Fig. 2). However, the differential expression of these genes defines no less than six photoreceptor subtypes and possibly five ommatidial subtypes [7]. For the purposes of this review, it is sufficient to focus on the yellow and pale subtypes of ommatidia, which fill most of the retina (Fig. 1 and 2). All Drosophila ommatidia, including yellow and pale ommatidia, express the LWS opsin Rh1 in the outer photoreceptors R1-R6. It thus is the differential expression of opsins Rh3-Rh6 in the inner photoreceptors R7 and R8 that distinguishes the ommatidial subtypes [9]. Yellow ommatidia express the SWS-UV opsin Rh4 in the R7 photoreceptor and the green-sensitive $R h 6$ in the R8 photoreceptors, while pale ommatidia express the SWS-UV opsin Rh3 in the R7 photoreceptor and SWS-B opsin Rh5 in the R8 photoreceptor (Fig. 2). Together, this translates into five opsin-defined photoreceptor subtypes in the yellow and pale ommatidia of the Drosophila eye: outer photoreceptors (Rh1positive), pale R7s (Rh3-positive), yellow R7s (Rh4-positive), pale R8s (Rh5-positive) and yellow R8s (Rh6-positive). 
Friedrich et al.: Compound eye evolution

The role of this complex mosaic of photoreceptor subtype-specific opsin expression in the visual ecology of Drosophila remains poorly understood [25,26]. At the physiological level, it has been established that different eye regions and distinct ommatidial subtypes serve specialized visual tasks. This includes the detection of polarized light in specific areas of the eye $[27,28]$, the achromatic tracking of motion by the monochromatic outer photoreceptors R1-R6 $[29,30]$, and the discrimination of colors by the spectrally diverse inner photoreceptors R7 and R8 [29,30]. Interestingly, recent work has demonstrated crosstalk between these motion and color vision systems [30-32]. Moreover, there is evidence that the contribution of outer and inner photoreceptors to motion vs color vision varies across species [33]. Given this complexity and diversity of pancrustacean compound eyes, it is fascinating to note the deep conservation of the basic outer vs inner photoreceptor subfates $[8,9]$. As described below, the same can now also be stated regarding the transcriptional mechanisms that generate the retinal mosaics of pancrustacean compound eyes.

\section{Conserved default activation of SWS opsin expression by Otd}

The complexity of differential opsin expression in the Drosophila compound eye has served as an efficient system for studying the transcriptional mechanisms involved in differential gene expression during cell fate differentiation. These efforts uncovered a surprisingly simple core logic of the gene regulatory network that controls the photoreceptor subtype-specific expression of opsin genes $R h 3, R h 5$, and Rh6 (Fig. 3 and 4) [10]. Each of these genes, while highly restricted to specific inner photoreceptors in the mature eye, has the default potential to be activated in all eight photoreceptors within an ommatidium. Thus, instead of photoreceptorspecific gene activation, it is transcriptional repression in incompatible photoreceptor subtypes that restricts opsin expression to the correct photoreceptor subtype.

The default activation and its transcriptional facilitators have been been found to be deeply conserved in Tribolium and Daphnia. The default activation of the Drosophila SWS opsins $R h 3$ and $R h 5$ is mediated through direct activation by Otd (Orthodenticle) [34], the single member of the Otx K50 homeodomain transcription factor family in Drosophila (Fig. 3) [34]. The ancientness of this factor is amply reflected in the level of its complex pleiotropic 
Friedrich et al.: Compound eye evolution

involvement in neurogenesis, including photoreceptor subtype specification. In the eye, in addition to promoting SWS opsin expression (Fig. 3 and 4), Otd promotes the pale R8 photoreceptor fate through regulation of the Hippo signaling pathway [35], indirectly represses $R h 3, R h 5$, and $R h 6$ in outer and yR7 photoreceptors by promoting the expression of the transcriptional repressor Defective proventriculus (Dve) [34,36], and indirectly represses $R h 6$ in the pale R8 photoreceptors by activating the large Maf transcription factor Traffic Jam (Tj) [35] . The sum of these Otd-involving mechanisms ultimately filters Otd-mediated default activation of opsins $R h 3$ and $R h 5$ to specific expression in the pale subsets of R7 and R8 photoreceptors (Fig. 3).

In addition to this extraordinary pleiotropy, the challenge of probing for evolutionary conservation of Otd-dependent functions has been exacerbated by the convoluted orthology relationships between the Otx factors of Drosophila, Tribolium, and Daphnia [22]. Both Daphnia and Tribolium possess two otd genes due to apparently independent duplications, while Drosophila has lost one otd homolog. Notwithstanding this dynamic gene family history, select otd homologs from Tribolium and Daphnia can rescue the activation of the SWS-UV opsin Rh3 in Drosophila pR7 photoreceptors [22]. Moreover, one of the Tribolium otd homologs (otd2) rescues the activation of the SWS-B opsin $R h 5$ [22]. Further documenting a conserved function of Otd factors in SWS-UV activation, knockdown of the Tribolium otd genes revealed that the otd2 paralog is essential for the expression of SWS-UV opsin in Tribolium [22]. Intriguingly, three K50 Otd candidate binding sites can be detected in the upstream region of the Tribolium SWS-UV opsin gene (A.Z. personal observation). This suggests that the SWS-UV opsin gene is directly activated by Tribolium Otd2, equivalent to the activation of SWS-UV opsin Rh3 by Otd in Drosophila (Fig. 3). Of further note, the downregulation of Tribolium Otd2 does not affect the activation of the Tribolium LWS opsin [22]. This is consistent with the Otd-specific activation of SWS opsins in Drosophila (Fig. 3) and speaks for an evolutionarily conserved split in the transcriptional default activation of SWS vs LWS opsin genes by Otd and a second factor, which has been identified as well.

\section{Conserved default activation of LWS opsin by Pph13}


Friedrich et al.: Compound eye evolution

The photoreceptor-wide transcriptional default activation of the green-sensitive LWS opsin Rh6 in the Drosophila eye is provided by the Aristaless-related (Arx) homeodomain transcription factor PvuII-PstI homology 13 (Pph13) [37]. Similar to the case of Otd and the Rh3 and Rh5 opsins, this Pph13-mediated default activation is filtered to the selective expression of Rh6 specifically in yellow R8 photoreceptors through concomitant transcriptional repression in the incompatible photoreceptor fates (Fig. 4). This involves the transcription factor Dve in outer photoreceptors R1-R6 [34,36], the transcription factor Prospero (Pros) in R7 photoreceptors [38], and the transcription factor $\mathrm{Tj}$ in the pale R8 photoreceptors [35].

Recent studies in Daphnia and Tribolium reveal that the role of Pph13 as a default activator of LWS opsin expression is indeed also ancestral in pancrustacean eye development [22]. Circumstantial evidence for this function already existed, in part, based on the evolutionary conservation of the Pph13 binding site within a previously defined photoreceptor-specific cisregulatory element, called the Rhodopsin core sequence I (RCSI) or P3 site. This 11 bp long palindromic homeodomain binding site was identified based on its conserved presence in the promoters of all six Drosophila opsin genes (Fig. 3 and 4) [39,40] and drives reporter gene expression in all photoreceptors in the Drosophila compound eye [34,39,41]. The latter could also be demonstrated for transgenic moths and beetles [42-45], presenting the first evidence that a conserved photoreceptor-selective factor is responsible for the RCSI/P3 mediated transcriptional activation of opsin expression.

Consistent with this role, Pph13 is highly restricted to photoreceptors in Drosophila, recognizes a portion of the RCSI/P3 site present in the RCSI/P3 reporter constructs, and is essential for mediating RCSI/P3 reporter gene expression in Drosophila [37]. Further analysis of Pph13-dependent binding properties, however, revealed higher binding affinity to a specific subset of of RCSI/P3 sites - that found in the LWS opsin Rh6, rather than those in the SWS opsins Rh3 and Rh5 in Drosophila. Consistent with this specificity for the Rh6 opsin, mutations in Pph13 lead to loss of Rh6 expression in the Drosophila compound eye, while Rh3 and Rh5 remain unaffected [37].

The recent studies in Tribolium and Daphnia have uncovered putative RCSI elements with Pph13 binding sites in a subgroup of the Daphnia LWS opsin gene regulatory sequences (the B lineage) and in both of the two opsin genes of Tribolium [22]. Similar to the situation in 
Friedrich et al.: Compound eye evolution

Drosophila, however, Tribolium Pph13 protein binds to the RCSI site of the LWS opsin with higher affinity than the RCSI site of the SWS-UV opsin [22]. Demonstrating a functionally conserved capacity of Pph13 in LWS opsin activation, both Tribolium and Daphnia Pph13 specifically rescue expression of the LWS Rh6 opsin in Drosophila mutants lacking endogenous Pph13 activity [22]. Furthermore, reducing Pph13 in Tribolium diminishes LWS but not SWSUV transcript levels, thus mirroring the misregulation of opsin expression in Pph13 mutant Drosophila eyes. Taken together, these findings identify Pph13 as an ancestral activator of LWS opsin expression in the pancrustacean retina through an LWS opsin-restricted RCSI site (Fig. 4).

\section{Conserved cooperativity of Otd and Pph13 in the transcriptional activation of rhabdomere formation}

In addition to their roles in opsin regulation, Otd and Pph13 are also both required for the proper formation of the opsin-containing rhabdomere compartment of photoreceptor cells $[37,46]$. Rhabdomeres form through initial expansion of the apical photoreceptor membrane into densely packed microvillar stacks that eventually extend along the proximodistal axis of the mature photoreceptor [47]. Drosophila eyes mutant for otd or Pph13 exhibit partial defects in rhabdomere morphogenesis $[48,49]$, while eyes mutant for both factors are completely void of rhabdomere structures [37]. Thus, in the Drosophila eye, otd and Pph13 function additively to regulate rhabdomere formation.

Comparative studies in Tribolium and Daphnia have now established that this involvement of otd and Pph13 in rhabdomere formation is likewise ancient for the pancrustacean eye [22]. Misexpression of both of the otd genes of Tribolium and the Daphnia otd1 homolog exhibit equivalent capacities to rescue otd mutant rhabdomeres in Drosophila. Similarly, expression of either the Tribolium or Daphnia Pph13 homologs in Drosophila Pph13 mutant photoreceptors rescues rhabdomere morphology close to wild type organization.

As a final demonstration, downregulating the otd orthologs or Pph13 abolishes rhabdomere formation in Tribolium, documenting the conserved involvement of these factors for rhabdomere formation in both flies and beetles [22]. 


\section{A developmental model of photoreceptor subtype evolution}

The roles of Otd and Pph13 in both differential opsin regulation and rhabdomere formation are likely no coincidence. Besides harvesting light, opsins also represent structural components of the rhabdomere, which is documented by the fact that reduced opsin expression levels leads to a failure in rhabdomere development [50,51]. This link leads to an integrated model of the evolution of rhabdomere structure and differential opsin expression in the ancestral pancrustacean retina (Fig. 5). The role of Otd in the transcriptional activation of photosensitive protein components likely preceded the pancrustacean ancestor. This conjecture is based on the involvement of Otx factors in the development of the ciliary photoreceptors of vertebrates, the capacity of vertebrate Otx factors to rescue Otd functions in the Drosophila eye, and the multilayered master regulatory roles of Otd in pancrustacean photoreceptor differentiation $[22,34,35,37,46,48,52,53]$. It is therefore reasonable to assume that an ancestral Otx homolog was the regulator of the prototype photosensitive membrane compartment that harvested light through opsins in the earliest animal photoreceptors.

The joining of Pph13 at a later stage of rhabdomeric photoreceptor evolution follows from the absence of Pph13 orthologs outside protostomes, which suggests a younger evolutionary age, in line with the exceptionally specific function of this factor to photoreceptors. It is thus tempting to speculate that Pph13 joined Otd in the upstream activation of rhabdomererelated genes during the evolution of a more elaborate rhabdomere organization that required extended cis-regulatory input. During this transition, Pph13 likely acquired the role of the LWS opsin expression activator through recognition of a subset of RCS/P3 sites, while Otd retained its ancestral role in activating opsin genes for the SWS opsin precursor. Consistent with the findings of a recent comprehensive analysis of pancrustacean opsin gene family evolution [54], the latter likely subsequently gave rise to the SWS-B and SWS-UV opsin subfamilies in the pancrustacean retina, both of which inherited the dependence on activation by Otd. This division of labor might have been the beginning of differential opsin expression in photoreceptor subtypes, which experienced further refinements in different lineages, likely through repressor mechanisms like those that shape the retinal mosaic of Drosophila. 
Friedrich et al.: Compound eye evolution

Besides explaining the complementary roles of Otd and Pph13 in opsin regulation and rhabdomere development, this model provides an evolutionary explanation for the default activation of opsins through Otd and Pph13 in the Drosophila retina. In further support of the model, Pph13-specific RCSI sites have been found in the orthologs of the photoreceptor-specific G protein $\beta$-subunit 76C gene (Gß76C) in Drosophila, Tribolium and Daphnia [22], revealing an extended range of conserved rhabdomeric target genes of Pph13 [49]. Similarly, a broader range of putative direct Otd target genes with rhabdomere-specific functions such as Arrestin 2 and Calphotin has been identified in Drosophila [55].

\section{Perspectives}

The model outlined above has several predictions inviting future studies. One obvious prediction is the conservation of the Pph13 and Otd opsin default activation cassettes in other pancrustacean compound eyes. Interestingly, a recent study on RCSI/P3 reporter gene expression in the butterfly species Bicyclus anynana concluded that Pph13 is absent from Lepidoptera [56]. Indeed, preliminary analysis confirms the absence of detectable Pph13 orthologs in most lepidopteran genome and transcriptome databases. The Glanville fritillary Melitaea cinxia whole genome shotgun sequence contig 12241, however, contains the coding sequence for an unambiguous Pph13 homeodomain (M.F., personal observation). Pph13 is thus likely also conserved in Lepidoptera and may have escaped detection in other moth and butterfly species so far due to the fact that the extent of its conserved sequence is confined to the homeodomain. In support of this speculation, the detection of Pph13 in the Daphnia genome also required targeted effort [22].

The evolutionary developmental model of photoreceptor subtype diversification presented here further predicts the conservation of Otd as the default activator specifically of SWS opsins vs conservation of Pph13 as default activator of LWS opsins. Given the efficient detectability of Otd- and Pph13-specific binding sites and the compact organization of the cisregulatory DNA of opsin genes, this prediction can be tested by bioinformatic studies in additional pancrustacean species. This objective will be aided by a new generation of binding 
Friedrich et al.: Compound eye evolution

site prediction programs [57-59]. The same tools should be further valuable for testing the postulated direct regulation of structural rhabdomere protein genes by Otd and Pph13 [22,55].

Evolutionary conservation can also be hypothesized for the repressive mechanisms that restrict the execution of the photoreceptor-wide default activities of Pph13 and Otd to photoreceptor subtypes. One such candidate mechanism is the repression of default LWS opsin expression in R7 photoreceptors by Pros (Fig. 4). The loss of this single input is an attractive explanation for the exceptional co-expression LWS and SWS-UV opsin in the R7 photoreceptors of the Tribolium eye (Fig. 2 and 4).

The RNAi knockdown approach, which has been highly efficient for characterizing gene functions [60], has recently been complemented by CRISPR-mediated gene knockout protocols in Tribolium and Daphnia [61,62]. This expanded range of genetic interference approaches can be expected to expedite the functional analysis of additional regulators in these models of pancrustacean compound eye development. The resulting insights are likely to benefit the investigation of other remarkable visual systems in the Pancrustacea such as recently characterized in dragonflies and stomatopod Crustacea, which display exceptional ommatidial diversity [63-66].

Compound eyes are also ancestral body plan components of the other two major arthropod subphyla - Chelicerates and Myriapods. It is therefore equally exciting to note a new generation of molecular developmental studies in the structurally and evolutionarily complex visual systems of chelicerates [67-69], opening further testing grounds for examining the conserved roles of Otd and Pph13 in photoreceptor differentiation.

\section{ACKNOWLEDGEMENTS}

We thank Cassandra Extavour and Leslie Pick for the invitation to contribute this review, Riyue Bao for help with compiling information on transcription factor binding sites in the regulatory DNA of Drosophila opsin genes, Donna Kashian for the picture of Daphnia magna, and the two anonymous reviewers for excellent comments. This work was supported by funds from NIH grant EY022687 (TAC), and NSF grants IOS-0951886 (MF) and IOS-1353267 (ACZ). 


\section{REFERENCES:}

1. $\quad$ Land MF, Nilsson DE: Animal Eyes. OUP Oxford; 2012.

2. Nilsson D-E: Eye evolution and its functional basis. Vis. Neurosci. 2013, 30:5-20.

3. Lee MSY, Jago JB, García-Bellido DC, Edgecombe GD, Gehling JG, Paterson JR: Modern optics in exceptionally preserved eyes of Early Cambrian arthropods from Australia. Nature 2011, 474:631-634.

4. Paterson JR, García-Bellido DC, Lee MSY, Brock GA, Jago JB, Edgecombe GD: Acute vision in the giant Cambrian predator Anomalocaris and the origin of compound eyes. Nature 2011, 480:237-240.

5. Charlton-Perkins M, Cook TA: Building a fly eye: Terminal differentiation events of the retina, corneal jens, and pigmented epithelia. Curr. Top. Dev. Biol. 2010, 93:129-173.

6. Briscoe AD, Chittka L: The evolution of color vision in insects. Annu. Rev. Entomol. 2001, 46:471-510.

7. Wernet MF, Perry MW, Desplan C: The evolutionary diversity of insect retinal mosaics: common design principles and emerging molecular logic. Trends Genet. 2015, 31:316-328.

8. Buschbeck EK, Friedrich M: Evolution of insect eyes: tales of ancient heritage, deconstruction, reconstruction, remodeling, and recycling. Evolution: Education and Outreach 2008, [no volume].

9. Melzer RR, Michalke C, Smola U: Walking on insect paths? Early ommatidial development in the compound eye of the ancestral crustacean, Triops cancriformis. Naturwissenschaften 2000, 87:308-311.

10. Johnston RJ Jr: Lessons about terminal differentiation from the specification of color-detecting photoreceptors in the Drosophila retina. Ann. N. Y. Acad. Sci. 2013, 1293:33-44.

$(\bullet)$ Integrated review of recently characterized differential opsin expression patterns in the fruit fly retina with respect to ecological specialization and evolution cis-regulatory control of opsin gene expression.

11. Rister J, Desplan C, Vasiliauskas D: Establishing and maintaining gene expression patterns: insights from sensory receptor patterning. Development 2013, 140:493-503.

12. Tribolium Genome Sequencing Consortium, Richards S, Gibbs RA, Weinstock GM, Brown SJ, Denell R, Beeman RW, Gibbs R, Beeman RW, Brown SJ, et al.: The genome of the model beetle and pest Tribolium castaneum. Nature 2008, 452:949-955.

13. Friedrich M, Benzer S: Divergent decapentaplegic expression patterns in compound eye development and the evolution of insect metamorphosis. J. Exp. Zool. 2000, 288:39-55. 
Friedrich et al.: Compound eye evolution

14. Henze MJ, Dannenhauer K, Kohler M, Labhart T, Gesemann M: Opsin evolution and expression in arthropod compound eyes and ocelli: insights from the cricket Gryllus bimaculatus. BMC Evol. Biol. 2012, 12:163.

15. Takagi A, Kurita K, Terasawa T, Nakamura T, Bando T, Moriyama Y, Mito T, Noji S, Ohuchi H: Functional analysis of the role of eyes absent and sine oculis in the developing eye of the cricket Gryllus bimaculatus. Dev. Growth Differ. 2012, 54:227-240.

16. Colbourne JK, Pfrender ME, Gilbert D, Thomas WK, Tucker A, Oakley TH, Tokishita S, Aerts A, Arnold GJ, Basu MK, et al.: The ecoresponsive genome of Daphnia pulex. Science 2011, 331:555561.

17. Friedrich M, Rambold I, Melzer RR: The early stages of ommatidial development in the flour beetle Tribolium castaneum (Coleoptera; Tenebrionidae). Dev. Genes Evol. 1996, 206:136-146.

18. Wolff T, Ready DF: The beginning of pattern formation in the Drosophila compound eye: the morphogenetic furrow and the second mitotic wave. Development 1991, 113:841-850.

19. Smith KC, Macagno ER: UV photoreceptors in the compound eye of Daphnia magna (Crustacea, Branchiopoda). A fourth spectral class in single ommatidia. Journal of Comparative Physiology A 1990, 166:597-606.

20. Mazzoni EO, Desplan C, Celik A: “One receptor” rules in sensory neurons. Dev. Neurosci. 2004, 26:388-395.

21. Jackowska M, Bao R, Liu Z, McDonald EC, Cook TA, Friedrich M: Genomic and gene regulatory signatures of cryptozoic adaptation: Loss of blue sensitive photoreceptors through expansion of long wavelength-opsin expression in the red flour beetle Tribolium castaneum. Front. Zool. 2007, 4:24.

22. Mahato S, Morita S, Tucker AE, Liang X, Jackowska M, Friedrich M, Shiga Y, Zelhof AC: Common transcriptional mechanisms for visual photoreceptor cell differentiation among Pancrustaceans. PLoS Genet. 2014, 10:e1004484.

(••) Comparative analysis of Otd and Pph13 expression and function during photoreceptor differentiation in the compound eyes of Drosophila, Tribolium, and Daphnia. Represents the first molecular analysis of compound eye development in Daphnia. Reports evidence that the cooperative involvement of Otd and Pph13 in the regulation of opsin expression and rhabdomere formation dates back to the last ancestor of the Pancrustacea and is functionally conserved between Drosophila, Tribolium, and Daphnia.

23. Brandon CS: The evolutionary biology of vision in Daphnia. 2015, [no volume].

24. Schehr RS: Spectral sensitivities of anatomically identified photoreceptors in the compound eye of Daphnia magna. 1984, [no volume].

25. Borst A: Drosophila’s view on insect vision. Curr. Biol. 2009, 19:R36-47.

26. Lunau K: Visual ecology of flies with particular reference to colour vision and colour preferences. J. Comp. Physiol. A Neuroethol. Sens. Neural Behav. Physiol. 2014, 200:497-512. 
Friedrich et al.: Compound eye evolution

$(\bullet)$ Up to date comprehensive review of color vision physiology, models, and behavioral evidence in brachyceran Diptera.

27. Wernet MF, Velez MM, Clark DA, Baumann-Klausener F, Brown JR, Klovstad M, Labhart T, Clandinin TR: Genetic dissection reveals two separate retinal substrates for polarization vision in Drosophila. Curr. Biol. 2012, 22:12-20.

$(\bullet)$ First genetic demonstration of polarized light detection capability in both the dorsal and ventral eye of Drosophila demonstrating that the dorsal sensitivity involves the inner photoreceptors R7 and R8 while the ventral sensitivity involves both outer and inner photoreceptors.

28. Fortini ME, Rubin GM: The optic lobe projection pattern of polarization-sensitive photoreceptor cells in Drosophila melanogaster. Cell Tissue Res. 1991, 265:185-191.

29. Yamaguchi S, Wolf $\mathrm{R}$, Desplan $\mathrm{C}$, Heisenberg M: Motion vision is independent of color in Drosophila. Proc. Natl. Acad. Sci. U. S. A. 2008, 105:4910-4915.

30. Yamaguchi S, Desplan C, Heisenberg M: Contribution of photoreceptor subtypes to spectral wavelength preference in Drosophila. Proc. Natl. Acad. Sci. U. S. A. 2010, 107:5634-5639.

31. Wardill TJ, List O, Li X, Dongre S, McCulloch M, Ting C-Y, O’Kane CJ, Tang S, Lee C-H, Hardie RC, et al.: Multiple spectral inputs improve motion discrimination in the Drosophila visual system. Science 2012, 336:925-931.

$(\bullet)$ Electrophysiological, optical, and behavioral demonstration that motion detection is in part also mediated by the inner photoreceptors in addition to the outer photoreceptors in Drosophila.

32. Schnaitmann C, Garbers C, Wachtler T, Tanimoto H: Color discrimination with broadband photoreceptors. Curr. Biol. 2013, 23:2375-2382.

(•) Computational and behavioral demonstration that color vision is also partly mediated by the otherwise motion-detection specific outer photoreceptors in addition to the inner photoreceptors in Drosophila.

33. Friedrich M, Wood EJ, Wu M: Developmental evolution of the insect retina: insights from standardized numbering of homologous photoreceptors. J. Exp. Zool. B Mol. Dev. Evol. 2011, 316:484-499.

34. Tahayato A, Sonneville R, Pichaud F, Wernet MF, Papatsenko D, Beaufils P, Cook T, Desplan C: Otd/Crx, a dual regulator for the specification of ommatidia subtypes in the Drosophila retina. Dev. Cell 2003, 5:391-402.

35. Jukam D, Xie B, Rister J, Terrell D, Charlton-Perkins M, Pistillo D, Gebelein B, Desplan C, Cook T: Opposite feedbacks in the Hippo pathway for growth control and neural fate. Science 2013, 342:1238016.

$(\bullet)$ Reports an evolutionarily conserved regulatory module for generating photoreceptor diversity that involves Otd/OTX and the large MAF transcription factor Traffic jam/NRL, and the inhibition of this function by the tissue growth regulatory Hippo signaling pathway. 
Friedrich et al.: Compound eye evolution

36. Johnston RJ Jr, Otake Y, Sood P, Vogt N, Behnia R, Vasiliauskas D, McDonald E, Xie B, Koenig S, Wolf R, et al.: Interlocked feedforward loops control cell-type-specific Rhodopsin expression in the Drosophila eye. Cell 2011, 145:956-968.

37. Mishra M, Oke A, Lebel C, McDonald EC, Plummer Z, Cook TA, Zelhof AC: Pph13 and orthodenticle define a dual regulatory pathway for photoreceptor cell morphogenesis and function. Development 2010, 137:2895-2904.

38. Cook T, Pichaud F, Sonneville R, Papatsenko D, Desplan C: Distinction between color photoreceptor cell fates is controlled by Prospero in Drosophila. Dev. Cell 2003, 4:853-864.

39. Fortini ME, Rubin GM: Analysis of cis-acting requirements of the $\mathbf{R h} 3$ and $\mathbf{R h} \mathbf{4}$ genes reveals a bipartite organization to rhodopsin promoters in Drosophila melanogaster. Genes Dev. 1990, 4:444-463.

40. Zuker CS, Mismer D, Hardy R, Rubin GM: Ectopic expression of a minor Drosophila opsin in the major photoreceptor cell class: distinguishing the role of primary receptor and cellular context. Cell 1988, 53:475-482.

41. Papatsenko D, Nazina A, Desplan C: A conserved regulatory element present in all Drosophila rhodopsin genes mediates Pax6 functions and participates in the fine-tuning of cell-specific expression. Mech. Dev. 2001, 101:143-153.

42. Sheng G, Thouvenot E, Schmucker D, Wilson DS, Desplan C: Direct regulation of rhodopsin 1 by Pax-6/eyeless in Drosophila: evidence for a conserved function in photoreceptors. Genes Dev. 1997, 11:1122-1131.

43. Horn C, Wimmer EA: A versatile vector set for animal transgenesis. Dev. Genes Evol. 2000, 210:630-637.

44. Thomas J-L, Da Rocha M, Besse A, Mauchamp B, Chavancy G: 3×P3-EGFP marker facilitates screening for transgenic silkworm Bombyx mori $\mathrm{L}$. from the embryonic stage onwards. Insect Biochem. Mol. Biol. 2002, 32:247-253.

45. Berghammer AJ, Klingler M, Wimmer EA: A universal marker for transgenic insects. Nature 1999, 402:370-371.

46. Fichelson P, Brigui A, Pichaud F: Orthodenticle and Kruppel homolog 1 regulate Drosophila photoreceptor maturation. Proc. Natl. Acad. Sci. U. S. A. 2012, 109:7893-7898.

$(\bullet)$ Reports that Otd cooperates with the ecdysone receptor (EcR) to repress Kruppel homolog 1 (Krh1) as a hormonally-controlled key step in the transcriptional activation of terminal photoreceptor differentiation.

47. Longley RL Jr, Ready DF: Integrins and the development of three-dimensional structure in the Drosophila compound eye. Dev. Biol. 1995, 171:415-433.

48. Vandendries ER, Johnson D, Reinke R: orthodenticle is required for photoreceptor cell development in the Drosophila eye. Dev. Biol. 1996, 173:243-255. 
Friedrich et al.: Compound eye evolution

49. Zelhof AC, Koundakjian E, Scully AL, Hardy RW, Pounds L: Mutation of the photoreceptor specific homeodomain gene Pph13 results in defects in phototransduction and rhabdomere morphogenesis. Development 2003, 130:4383-4392.

50. Kumar JP, Ready DF: Rhodopsin plays an essential structural role in Drosophila photoreceptor development. Development 1995, 121:4359-4370.

51. Kumar JP, Bowman J, O’Tousa JE, Ready DF: Rhodopsin replacement rescues photoreceptor structure during a critical developmental window. Dev. Biol. 1997, 188:43-47.

52. Mencarelli C, Pichaud F: Orthodenticle is required for the expression of principal recognition molecules that control axon targeting in the Drosophila retina. PLoS Genet. 2015, 11:e1005303.

$(\bullet)$ Reports a novel role of Otd in Drosophila terminal photoreceptor differentiation: (I) axon targeting in the outer R1-6 photoreceptors through the transcriptional regulation of the recognition proteins Flamingo (fmi) and Golden Goal (gogo) and (II) axon targeting in the inner R8 photoreceptors through regulating Flamingo and Golden Goal together with the cell adhesion molecule Capricious.

53. Terrell D, Xie B, Workman M, Mahato S, Zelhof A, Gebelein B, Cook T: OTX2 and CRX rescue overlapping and photoreceptor-specific functions in the Drosophila eye. Dev. Dyn. 2012, 241:215-228.

$(\bullet \bullet)$ Demonstrates that human OTX genes and their disease-associated alleles rescue Otd phenotypes in Drosophila photoreceptors, in both overlapping and distinct manners. Reveals the deep functional conservation of the Otd gene family in photoreceptor differentiation and the feasibility of using Drosophila for the molecular genetic analysis of disease-associated human Otx gene alleles.

54. Henze MJ, Oakley TH: The dynamic evolutionary history of pancrustacean eyes and opsins. Integr. Comp. Biol. 2015, doi:10.1093/icb/icv100.

$(\bullet)$ A new integrated analysis of the diversity of eye types and opsin genes in Pancrustacea that concludes that the last common ancestor of Pancrustacea possessed for opsin gene subfamilies (LW2, MW1, MW2, and SW), which experienced a dynamic history of changes in eye type-specific expression.

55. Ranade SS, Yang-Zhou D, Kong SW, McDonald EC, Cook TA, Pignoni F: Analysis of the Otddependent transcriptome supports the evolutionary conservation of CRX/OTX/OTD functions in flies and vertebrates. Dev. Biol. 2008, 315:521-534.

56. Gupta M Das, Chan SKS, Monteiro A: Natural loss of eyeless/Pax6 expression in eyes of Bicyclus anynana adult butterflies likely leads to exponential decrease of eye fluorescence in transgenics. PLoS One 2015, 10:e132882.

57. Potier D, Seyres D, Guichard C, Iche-Torres M, Aerts S, Herrmann C, Perrin L: Identification of cis-regulatory modules encoding temporal dynamics during development. BMC Genomics 2014, 15:534.

58. Naval-Sánchez M, Potier D, Haagen L, Sánchez M, Munck S, Van de Sande B, Casares F, Christiaens V, Aerts S: Comparative motif discovery combined with comparative 
Friedrich et al.: Compound eye evolution

transcriptomics yields accurate targetome and enhancer predictions. Genome Res. 2013, 23:7488.

59. Herrmann C, Van de Sande B, Potier D, Aerts S: i-cisTarget: an integrative genomics method for the prediction of regulatory features and cis-regulatory modules. Nucleic Acids Res. 2012, 40:e114.

60. Miller SC, Miyata K, Brown SJ, Tomoyasu Y: Dissecting systemic RNA interference in the red flour beetle Tribolium castaneum: parameters affecting the efficiency of RNAi. PLoS One 2012, 7:e47431.

61. Nakanishi T, Kato Y, Matsuura T, Watanabe H: CRISPR/Cas-mediated targeted mutagenesis in Daphnia magna. PLoS One 2014, 9:e98363.

$(\bullet)$ First functional analysis of eye development in a Crustacean, demonstrating the efficacy of producing knockout mutants in the water flea Daphnia magna by genome editing and comparing the severity of eye defects in eyeless knockout versus eyeless knockdown animals.

62. Gilles AF, Schinko JB, Averof M: Efficient CRISPR-mediated gene targeting and transgene replacement in the beetle Tribolium castaneum. Development 2015, 142:2832-2839.

$(\bullet)$ First demonstration and detailed analysis of genome editing by CRISPR approach in Tribolium.

63. Porter ML, Zhang Y, Desai S, Caldwell RL, Cronin TW: Evolution of anatomical and physiological specialization in the compound eyes of stomatopod crustaceans. J. Exp. Biol. 2010, 213:3473-3486.

64. Porter ML, Bok MJ, Robinson PR, Cronin TW: Molecular diversity of visual pigments in Stomatopoda (Crustacea). Vis. Neurosci. 2009, 26:255-265.

65. Porter ML, Speiser DI, Zaharoff AK, Caldwell RL, Cronin TW, Oakley TH: The evolution of complexity in the visual systems of stomatopods: insights from transcriptomics. Integr. Comp. Biol. 2013, 53:39-49.

(•) Transcriptome-wide characterization of vision -related genes in two additional stomatopod species, significantly extending the known repertoire of the exceptionally diverse opsin gene family in the highly visual stomatopods.

66. Futahashi R, Kawahara-Miki R, Kinoshita M, Yoshitake K, Yajima S, Arikawa K, Fukatsu T: Extraordinary diversity of visual opsin genes in dragonflies. Proc. Natl. Acad. Sci. U. S. A. 2015, 112:E1247-56.

$(\bullet)$ Reports and characterizes the expression of the unexpectedly large number of opsin genes in dragonflies.

67. Samadi L, Schmid A, Eriksson BJ: Differential expression of retinal determination genes in the principal and secondary eyes of Cupiennius salei Keyserling (1877). Evodevo 2015, 6:16.

$(\bullet)$ Reports the expression of Otx, Six, and Pax6 homologs in the developing visual system of the wandering spider Cupiennius salei. One of two recent studies that characterize for the first time the 
Friedrich et al.: Compound eye evolution

developing embryonic visual system of spiders.

68. Eriksson BJ, Fredman D, Steiner G: Characterisation and localisation of the opsin protein repertoire in the brain and retinas of a spider and an onychophoran. BMC Evol. Biol. 2013, 13:186.

69. Schomburg C, Turetzek N, Schacht MI, Schneider J, Kirfel P, Prpic N-M, Posnien N: Molecular characterization and embryonic origin of the eyes in the common house spider Parasteatoda tepidariorum. Evodevo 2015, 6:15.

$(\bullet)$ Reports the expression of Otx, Six, Pax6, Eyes absent, and Dachshund homologs in the developing visual system of common house spider Parasteatoda tepidariorum. One of two recent studies that characterize for the first time the developing embryonic visual system of spiders.

70. Xie B, Charlton-Perkins M, McDonald E, Gebelein B, Cook T: Senseless functions as a molecular switch for color photoreceptor differentiation in Drosophila. Development 2007, 134:4243-4253. 


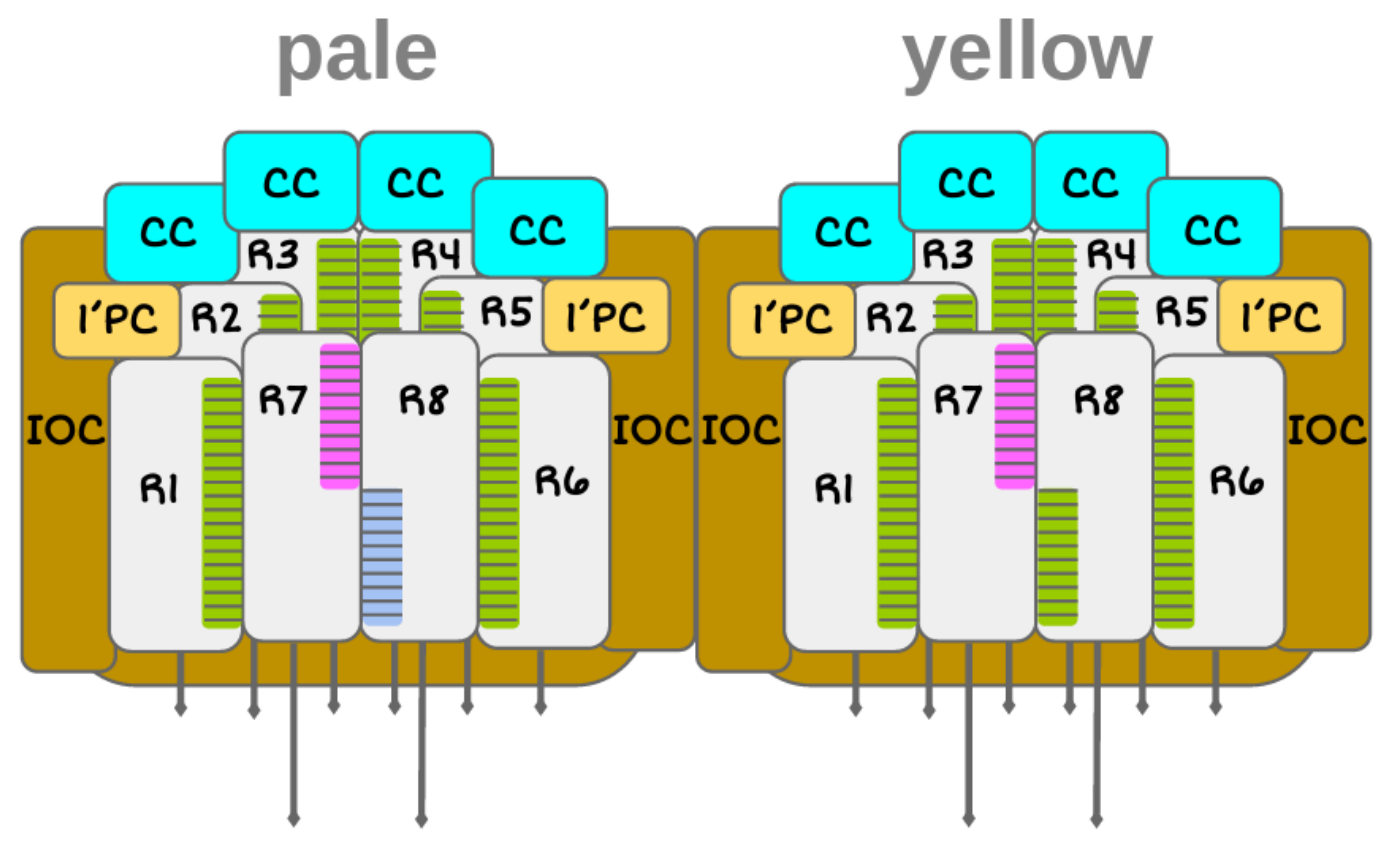

Fig. 1. Cellular organization of the ommatidium in the pancrustacean compound eye. Color code and abbreviations of cell types: Photoreceptors R1-R8 = light grey; Cone cells (CC) = turquoise; Primary pigment cells (1'PC) = yellow; Interommatidial cells (IOCs) = brown. The subcellular localization of the wavelength-specific opsin proteins in the photoreceptor rhabdomeres is indicated by green, blue, and magenta background shading. The examples above illustrate the difference between the pale and yellow subtype ommatidia in the Drosophila eye. In both subtypes, the outer photoreceptors R1-R6 express the LWS opsin Rh1. In pale ommatidia, the inner R7 photoreceptor expresses SWS-UV opsin Rh3 while the inner R8 photoreceptor expresses SWS-B opsin, Rh5. In yellow ommatidia, the R7 photoreceptor also expresses a SWS-UV opsin, Rh4, while the inner R8 photoreceptor expresses LWS opsin Rh6. The photoreceptor subtypes also differ by position, rhabdomere localization, and the depth of axonal projection in addition to the differential expression of opsin genes. The basic arrangements of both photoreceptor and accessory cells (cone cells, primary pigment cells, and interommatidial cells) as well as of inner and outer photoreceptor cell fates are highly conserved in pancrustacean compound eyes [33]. 


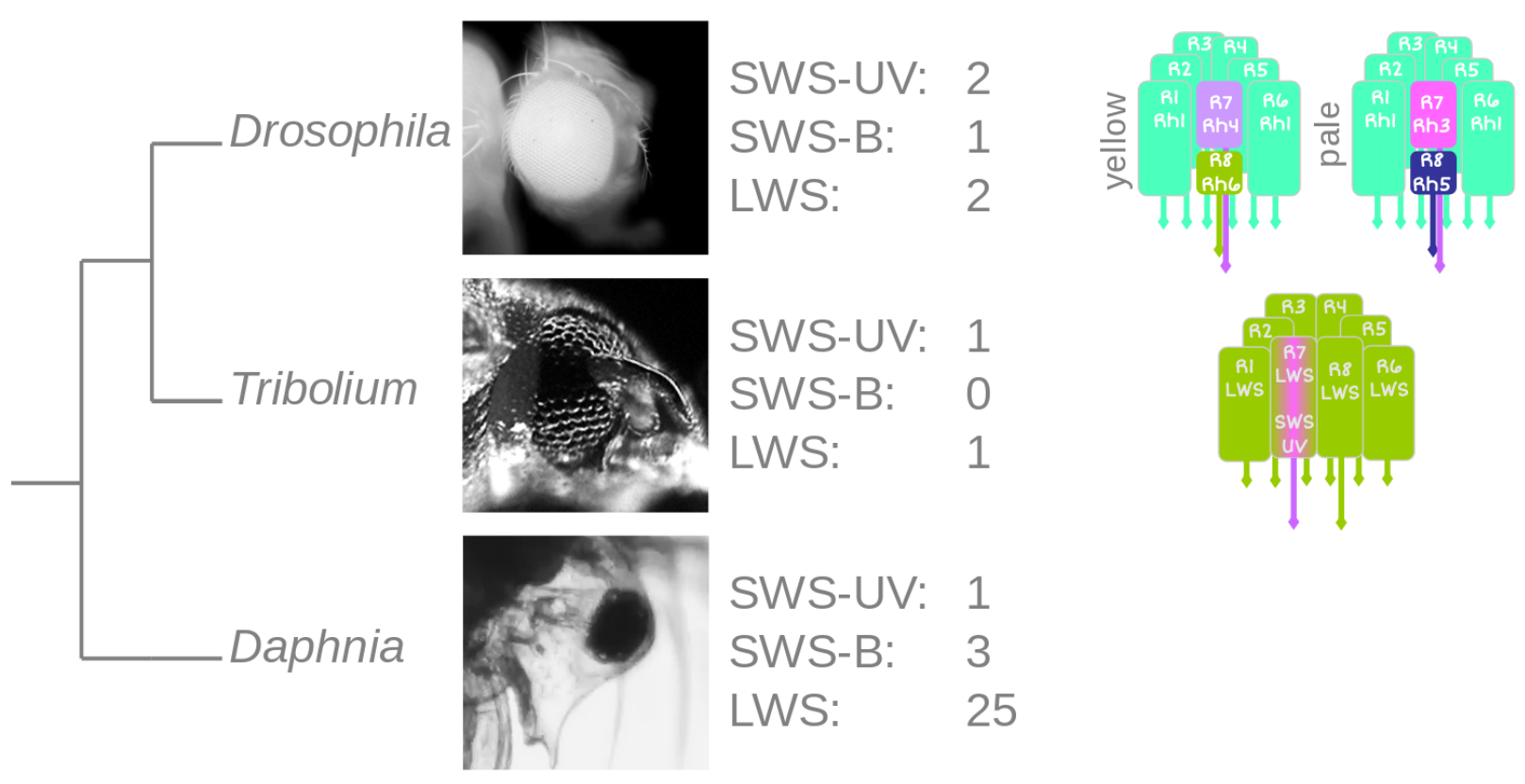

Fig. 2. Compound eye organization, ommatidial subtypes, and opsin gene repertoires in Drosophila, Tribolium, and Daphnia. See text for details on the differential expression of opsin paralogs in the ommatidia schematics shown for Drosophila and Tribolium. 
Otd

B

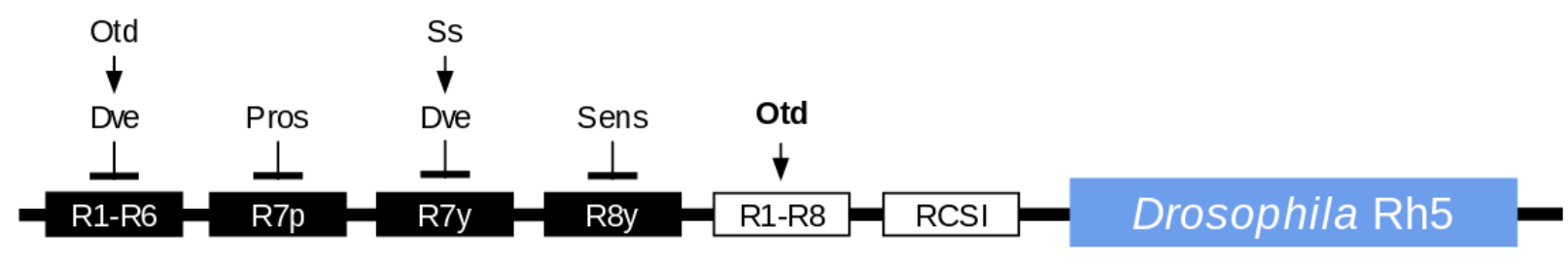

Fig. 3. Cis-regulatory evidence of the conserved role of Otd in the photoreceptor-wide transcriptional activation of SWS opsin expression. White boxes indicate sequence elements that bind transcriptional activators. Black boxes indicate sequence elements that bind transcriptional repressors. Bold gene names indicate functions that have been found conserved between Drosophila and Tribolium based on [22]. Arrangement of binding sites does not to scale with their relative positions in the native cis-regulatory DNAs. 
Friedrich et al.: Compound eye evolution
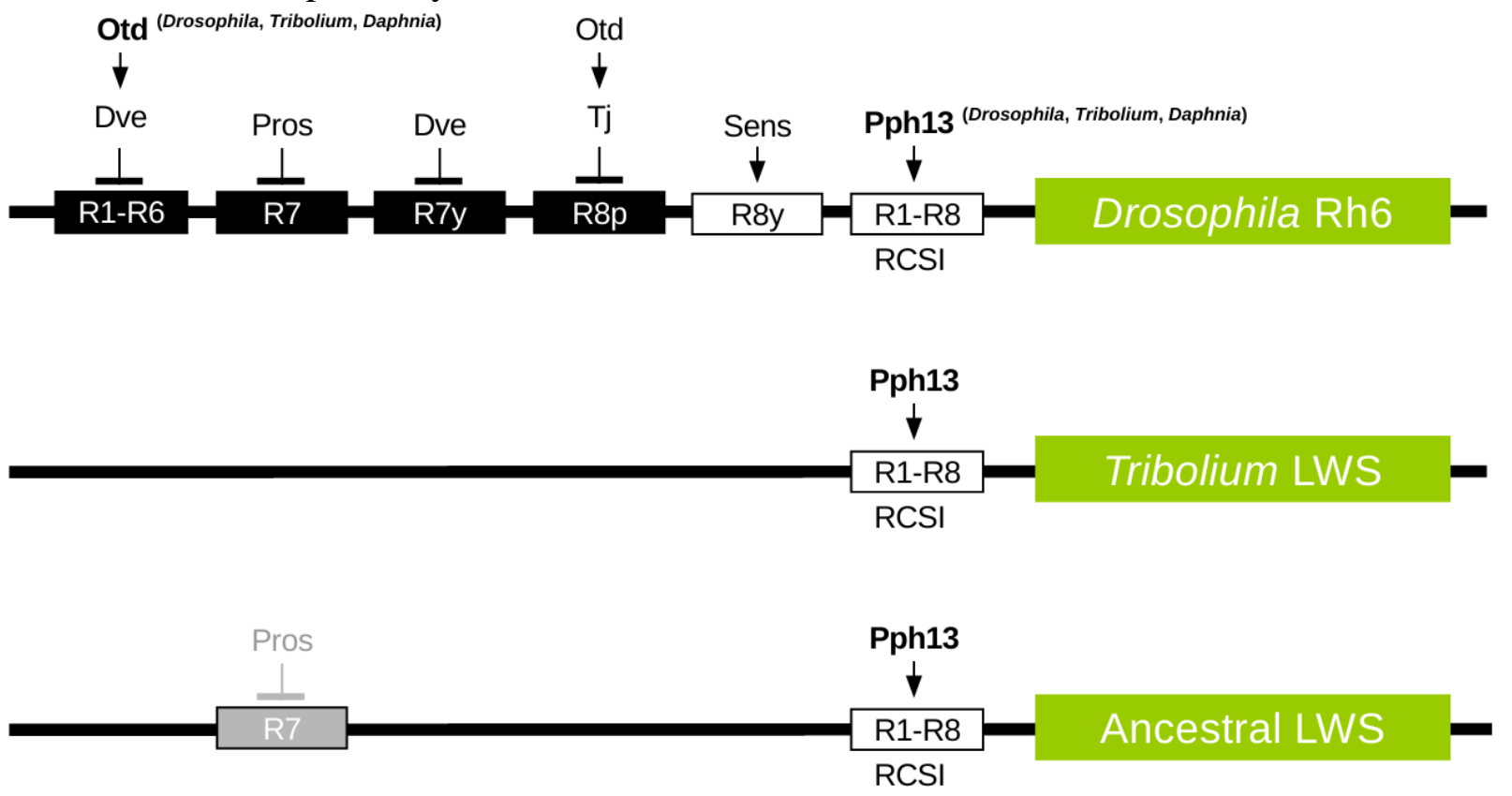

Fig. 4. Cis-regulatory evidence of the conserved role of Pph13 in the photoreceptor-wide transcriptional activation of LWS opsin expression. White boxes indicate sequence elements that bind transcriptional activators. Black boxes indicate sequence elements that bind transcriptional repressors. Grey box indicates presumed ancestral sequence element in the ancestral LWS opsin gene precursor hypothesized to be absent due to loss in Tribolium. Bold gene names indicate functions that have been demonstrated to be conserved between Drosophila, Tribolium, and Daphnia based on [22]. The capacity of Tribolium and Daphnia orthologs to rescue specific opsin regulation functions in Drosophila is indicated by superscripts.

Arrangement of binding sites does not equal their relative positions in the native cis-regulatory DNAs. Activating input from the additional transcription factor Senseless (Sens) in yellow R8 photoreceptors based on [70]. 


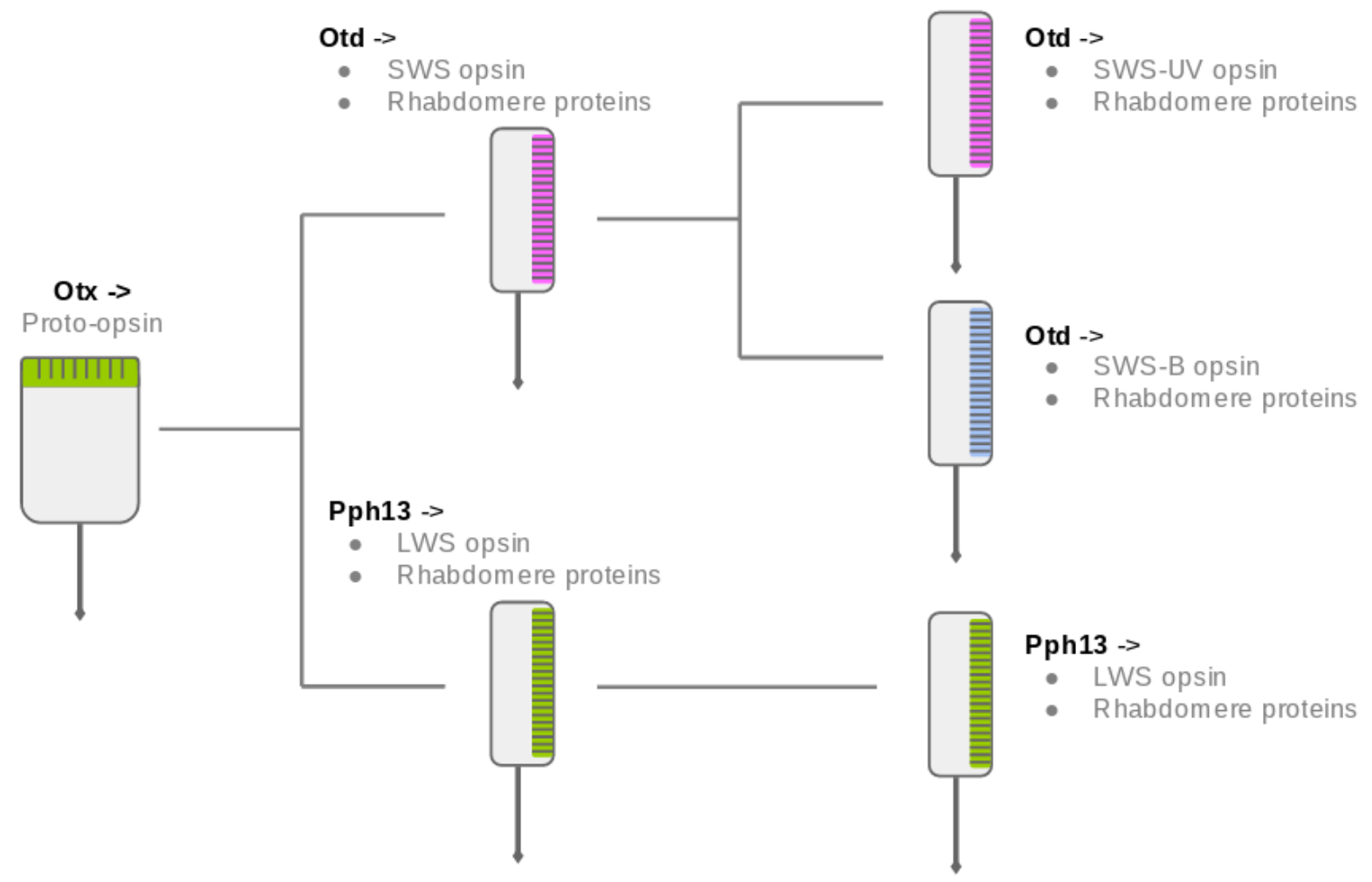

Fig. 5. A developmental model of pancrustacean photoreceptor subtype evolution through coordinated regulation of rhabdomeric proteins by Pph13 and Otd. Arrows represent direct transcriptional activation of target gene classes listed in grey. See text for further details. 